\title{
Tailored Waveform Generation in Mode-Locked Fiber Lasers by In-Cavity Pulse Shaper
}

\author{
Sonia Boscolo, ${ }^{1}$ Christophe Finot, ${ }^{2}$ and Periklis Petropoulos ${ }^{3}$ \\ ${ }^{1}$ Aston Institute of Photonic Technologies, Aston University, Birmingham B4 7ET, United Kingdom \\ ${ }^{2}$ Laboratoire Interdisciplinaire Carnot de Bourgogne, UMR 6303, BP 47870, 21078 Dijon Cedex, France \\ ${ }^{3}$ Optoelectronics Research Centre, University of Southampton, Highfield, Southampton SO17 1BJ, United Kingdom \\ s.a.boscolo@aston.ac.uk
}

\begin{abstract}
We numerically show the possibility of pulse shaping in a mode-locked fiber laser by inclusion of an amplitude-phase spectral filter into the laser cavity. Various advanced temporal waveforms are generated, including parabolic, flat-top and triangular pulses.
\end{abstract}

OCIS codes: $140.4050,320.5540,070.2615$.

\section{Introduction}

Passively mode-locked fiber laser technologies are fast growing solutions to provide stable, compact and cost-effective short pulse sources. In general, such lasers rely on nonlinear processes to achieve mode-locking, and major steps forward in pulse energy and peak power performance have been made with the recent discovery of new nonlinear mechanisms of pulse generation [1]. Thus far however, mode-locked fiber lasers have been relatively limited in terms of their reconfigurability, and achieving different output pulse characteristics generally involves significant changes to the physical layout of the cavity. The inclusion of a spectral pulse shaper into the cavity of a passively mode-locked fibre laser was recently demonstrated to enable wavelength and repetition rate tunability [2], and precise control of the cavity dispersion of the laser as well as to change the output pulse train from bright to dark pulses [3,4]. In this paper we take advantage of the amplitude and phase filtering ability of an in-cavity spectral pulse shaper to numerically show the possibility to achieve different pulse-shaping regimes of fundamental and practical interest in a mode-locked fiber laser [5].

\section{Operation principle and results}
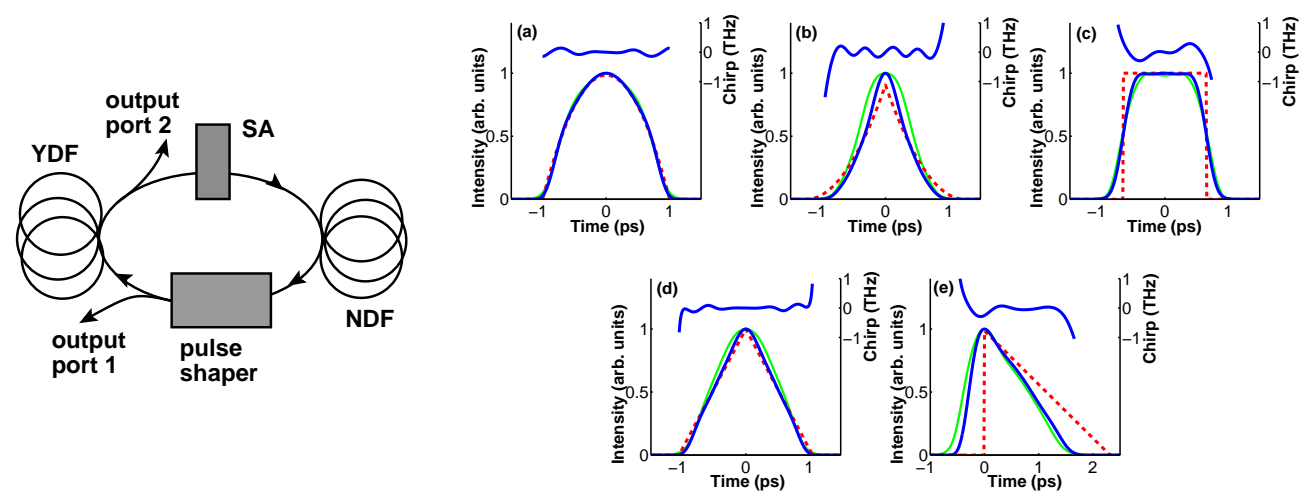

Fig. 1. Left: Schematic of the laser. Right: Temporal intensity and chirp profiles of the pulse after the pulse shaper (blue solid) for a (a) bright parabolic, (b) dark parabolic, (c) flat-top, (d) triangular and (e) saw-tooth shaping function. Also shown are the target intensity profiles (dashed red) and the profiles at the exit of the gain fiber (green).

The cavity configuration considered is a simple ring cavity (Fig. 1). The gain is provided by a $1 \mathrm{~m}$-long segment of ytterbium-doped fiber (YDF) with normal group-velocity dispersion (GVD), while $6 \mathrm{~m}$ of single-mode fiber with 
normal GVD acts as the nonlinear element of the cavity. A saturable absorber (SA) element follows the gain fiber, and an amplitude and phase filter is placed between the passive fiber and the amplifier to realize the pulse shaping. We will discuss details of the model during the conference presentation. The filter is modeled by the spectral response $\hat{H}(\omega)=$ $\hat{h}(\omega) \exp \left(i \beta_{2, \text { acc }} \omega^{2} / 2\right)$, where $\hat{h}(\omega)$ denotes the Fourier transform of a certain objective waveform function $h(t)$, and the spectral phase adds a specific amount of GVD $\beta_{2, \text { acc }}$ (in $\mathrm{ps}^{2}$ ) to the cavity which permits to control the net cavity dispersion. We consider five different amplitude functions in our study, which represent some examples of interesting waveform generations. We use a bright parabolic, a dark parabolic, a flat-top, a triangular and a saw-tooth pulse shaper with the respective functions: $h(t)=\sqrt{1-(t / \tau)^{2}} \theta(\tau-|t|) ; h(t)=(1-|t / \tau|) \theta(\tau-|t|) ; h(t)=\theta(\tau-|t|)$; $h(t)=\sqrt{1-|t / \tau|} \theta(\tau-|t|)$; and $h(t)=\sqrt{1-t / \tau}$ in $t \in[0, \tau], h(t)=0$ otherwise. Here $\theta(x)$ is the Heaviside function. Note that the spectral filter can be realized in a variety of ways, such as a fiber Bragg grating or even a programmable liquid crystal on silicon optical processor, which offers the additional advantage of being easily reconfigurable [6]; such devices are already commercially available.

The solutions obtained for the different amplitude profiles applied to the filter show that the desired pulse shapes are obtained at the output of the filter (Fig. 1). The generated parabolic and triangular pulses are nearly transform-limited, whereas the flat-top pulse features a small positive chirp. We can also see from Fig. 1 that, as opposed to the chirp profile targeted by the filter, the chirp profile of the generated pulses may exhibit a small ripple. This indicates that generating pulses with a well-defined phase profile by use of the method presented in this paper is not as straightforward. Figure 1 also highlights that the temporal intensity profile imparted by the filter onto the pulse is reasonably well preserved after the amplification stage, where more energetic pulses can be extracted from the cavity. The intra-cavity pulse evolution (not shown) revealed the following features common to all shaping regimes studied here. During the short amplification stage there is no strong reshaping (which confirms the results in Fig. 1), and that most of the nonlinear dynamics occur in the long passive fiber. The laser relies on rather strong nonlinearity combined with spectral filtering to stabilize the pulse in the cavity. The nonlinear evolution in the passive fiber is monotonic with the growth of temporal and spectral widths. The filter compensates for temporal and spectral broadening and cancels the large temporal phase accumulation in the fiber. Because the nonlinearly broadened spectrum of the pulse at the output of the passive fiber is significantly wider than the filter's spectral response and fairly flat within the filter bandwidth, it can be sliced without caring for the details of its structure. This is the enabling mechanism for the formation of pulses of any desired temporal shape at the output of the filter, determined by the filter's transfer function.

To gain a broader understanding of the laser dynamics, we investigated the effect of net dispersion on the laser response. The results revealed that the potential of tuning the output pulse temporal duration through in-cavity linear chirping is minimal. This stems from the filtering process being used, i.e., slicing of a much broader spectrum, and indicates that one should act directly on the waveform function of the filter to control the output pulse width. This result is not surprising, in fact, as a triangular, parabolic, or flat-top temporal profile is rather sensitive to group delay. Note that the net dispersion is controlled by adjusting the transfer function of the pulse shaper; therefore, no physical change in the cavity is required in order to change the output from one pulse shape to another, provided that a programmable pulse shaper is used.

\section{Conclusion}

We have numerically shown the operation of a passively mode-locked fiber laser in different pulse-shaping regimes, including bright and dark parabolic, flat-top, triangular and saw-tooth waveform generations, depending on the amplitude profile of an in-cavity spectral filter. Using a spectral filter based on a programmable optical processor, one would be able to easily reconfigure the operation of the laser with a computer interface. Our results are of particular relevance with the high interest in the generation of specialized pulse waveforms for applications in optical signal processing and manipulation.

\section{References}

1. W. H. Renninger, A. Chong, and F. W. Wise, IEEE J. Sel. Top. Quantum Electron. 18, 389-398 (2012).

2. J. Schröder, T. D. Vo, and B. J. Eggleton, Opt. Lett. 34, 3902-3904 (2009).

3. X. Yang et al., in CLEO 2013, OSA Techn. Dig. (OSA, 2013), paper CM1I.1.

4. J. Schröder et al., Opt. Express 18, 22715-22721 (2010).

5. S. Boscolo et al., Opt. Lett. 39, 438-441 (2014).

6. M. Roelens et al., J. Lightwave Technol. 26, 73-78 (2008). 